\title{
Un terrain vepse
}

Jean Léo Léonard et Ksenija Djordjević-Léonard

\section{(2) OpenEdition}

Journals

Édition électronique

URL : https://journals.openedition.org/efo/4376

DOI : $10.4000 /$ efo 4376

ISSN : 2275-1947

Éditeur

INALCO

\section{Édition imprimée}

Date de publication : 1 janvier 2014

ISBN : 978-2-343-05394-3

ISSN : 0071-2051

\section{Référence électronique}

Jean Léo Léonard et Ksenija Djordjević-Léonard, « Un terrain vepse », Études finno-ougriennes [En ligne], 46 | 2014, mis en ligne le 15 octobre 2015, consulté le 06 octobre 2022. URL : http:// journals.openedition.org/efo/4376 ; DOI : https://doi.org/10.4000/efo.4376

Ce document a été généré automatiquement le 6 octobre 2022.

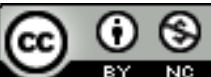

Creative Commons - Attribution - Pas d'Utilisation Commerciale 4.0 International - CC BY-NC 4.0 https://creativecommons.org/licenses/by-nc/4.0/ 


\title{
Un terrain vepse
}

\author{
Jean Léo Léonard et Ksenija Djordjević-Léonard
}

1 Suite à une première expérience de terrain en république de Mordovie en 2003 et 2004', qui a eu pour résultat de nombreuses publications et communications orales de la part des deux auteurs de la présente contribution ${ }^{2}$, nous avons organisé, à la fin de l'année 2012, un séjour de recherche et de terrain en république de Carélie; celui-ci commençait par une prise de contact avec des partenaires russes, spécialistes de langues finno-ougriennes et travaillant sur le vepse, langue sur laquelle a porté notre choix pour cette mission. Pour Jean Léo Léonard, c'était la réalisation d'un vieux «rêve » d'étudiant finno-ougriste - rêve alimenté, trente ans auparavant, par la lecture des grammaires historiques (Kettunen, 1922, 1960 ; Tunkelo, 1946) et des récits de voyage de Lauri Kettunen chez les Vepses méridionaux (Kettunen, 1945). À cette époque - dans les années 1980 - le terrain vepse n'était accessible qu'à de rares chercheurs occidentaux, surtout finlandais, ou à des chercheurs estoniens, comme Aime Kährik ou Lembit Vaba, de l'Institut de langue et de littérature ${ }^{3}$. Pour Ksenija Djordjević Léonard, le terrain vepse représentait une nouvelle expérience d'un microcosme finno-ougrien en Russie, dans la continuité et en contraste avec les deux précédentes missions en république de Mordovie, en 2003 et 2004. Pour les deux chercheurs, le vepse était donc un horizon plus proche que les langues de la Volga, à la fois plus familier et entièrement nouveau. Nous avions lu de nombreux témoignages qui convergeaient vers un profond pessimisme pour les perspectives de survie du vepse: villages abandonnés ou déserts, ou sans jeunesse et sans enfants, sans perspective d'avenir, envahis par les mauvaises herbes; population âgée comme dernier "sanctuaire» de la langue, mais ne trouvant plus personne à qui parler; économie rurale exsangue. Nous avions aussi entendu parler de locuteurs âgés ne parlant plus qu'une variété mixte de vepse, si mêlée de russe qu'aucun linguiste de l'époque glorieuse des néogrammairiens finlandais (Kettunen, Setälä, Tunkelo) n'en aurait voulu comme échantillon. Nous nous attendions au pire, non pas en termes d'accueil, mais en termes de vitalité de la langue. Seule ligne de fuite laissant de l'espace à quelque espoir : la thèse de Laura Siragusa sur les activistes vepses, que nous n'avons d'ailleurs pu consulter que la veille du départ. Mais là encore, la lecture pouvait aussi bien s'orienter vers une interprétation pessimiste qu'optimiste, selon le point de 
référence adopté. Le nôtre était nettement pessimiste. Nous verrons en quoi ce séjour nous a permis de nuancer cette vision des pratiques langagières actuelles du vepse.

2 Cette langue, bien qu'abondamment décrite par des spécialistes finnois ou russiens ${ }^{4}$, souvent à partir de recherches sur le terrain et de données de première main, est peu connue en France en dehors du cercle étroit de spécialistes du monde ouralien. Lorsque c'est le cas, il s'agit souvent de connaissances livresques, sans contact direct avec la réalité du terrain ni avec des locuteurs. Cependant, son importance pour la connaissance des structures linguistiques des langues du monde est telle qu'une recherche de terrain nous a semblé nécessaire, d'autant plus que la langue vepse se trouve aujourd'hui en situation de grande vulnérabilité, menacée de disparition à plus ou moins court terme $e^{5}$, et qu'en tant que linguistes, nous étions conscients que les conditions d'étude de cette langue ne seraient bientôt plus réunies. Dans ce qui suit, nous présenterons nos impressions de ce premier séjour en république de Carélie, à partir de nos observations personnelles et des informations collectées sur place, de la façon la plus objective possible, si tant est que l'on puisse parler d'objectivité dans un texte qui décrit un terrain et une réalité vus par nos yeux de chercheurs, avec toute la subjectivité qui caractérise une démarche de linguistique empirique allant à la rencontre non pas seulement des données, mais du milieu humain.

\section{Préparatifs}

3 Comment faire lorsqu'on ne connaît personne dans le monde académique carélien susceptible de nous aider dans nos démarches? Nos deux missions en Mordovie nous avaient appris à quel point le choix des partenaires officiels était important pour mener à bien une mission scientifique en Russie. Les différentes péripéties autour du choix des collaborateurs ont rendu notre première mission en Mordovie très éprouvante sur le plan psychologique ${ }^{6}$, et peuvent certainement expliquer notre grande réticence à travailler de nouveau sur les langues de Russie pendant pratiquement une décennie ${ }^{7}$.

L'occasion de nous intéresser au vepse nous a été fournie par notre implication dans un projet franco-russe coordonné par l'université de Bordeaux 3 et l'université de $\mathrm{Moscou}^{8}$, intitulé «Le besoin linguistique dans les marges linguistiques » et qui devait, s'il avait été accepté, se dérouler de 2012 à 2015. Dans ce projet centré sur la notion de besoin en relation avec une configuration-type de langues en situation minoritaire dans des marges linguistiques territoriales en Europe, notre rôle était de travailler précisément sur le vepse de Carélie, cette région faisant partie des terrains d'étude retenus, au même titre que l'Alsace en France par exemple. Hélas, alors que le projet avait été accepté dès 2011 par la Fondation scientifique de Russie pour les sciences humaines, l'aval du CNRS en France faisait défaut. Cependant, certains participants pressentis pour le projet avaient déjà commencé à réfléchir et à travailler sur les différents terrains définis pour l'étude. C'était le cas, par exemple, d'A. Viaut, de S. Moskvičeva et de S. Kovaleva qui ont commencé à travailler sur le carélien sans attendre les résultats de l'acceptation / du refus du projet en France ${ }^{9}$. Malgré l'absence de financement de la part du CNRS, ce sera également notre cas en 2013. Notre participation ponctuelle dans la phase d'élaboration du projet par les deux coordinateurs nous a permis d'obtenir du moins les premiers contacts sur place, à défaut de bénéficier de financements ${ }^{10}$ : Irma Mullonen, docteur en philologie, directrice de l'Institut de langue, littérature et histoire du Centre scientifique de Carélie 
(Académie des Sciences de Russie) et Svetlana Kovaleva, collaboratrice scientifique de ce même Institut, qui va s'avérer être le centre névralgique de toute recherche sur les langues finno-ougriennes de Carélie.

5 Les véritables préparatifs pour le séjour de terrain prévu en avril-mai 2013 ont commencé fin décembre 2012, lorsque nous avons reçu une réponse positive du Centre scientifique de Carélie. L'invitation officielle est arrivée en février, nous avons eu nos visas en mars ${ }^{11}$, et nous avons pu partir pour la Russie, comme prévu, en avril 2013. Ce voyage ne s'est pas déroulé sans appréhension, tant il est vrai que les images qui nous proviennent de la Russie par le biais des médias ne sont pas toujours rassurantes, en ce qui concerne la liberté de mouvement et d'expression. Cela peut prêter à sourire, mais la veille du départ nous en étions même à nous demander si dans nos écrits nous n'avions pas été trop critiques vis-à-vis de la Russie - critiques qui auraient pu, qui sait, nous attirer des ennuis une fois sur place. Il n'en fut rien et nous dûmes reconnaitre par devers nous, après coup, qu'une part de nos angoisses de la mission en Mordovie de 2003 pouvaient encore faire surface en nous. Partout où nous sommes allés, nous avons été très bien accueillis. Les détails quelque peu désagréables de notre premier séjour en Mordovie (confinement dans des villages, surveillance constante, manque de liberté de décision, lourdeur bureaucratique...) ont vite laissé la place au sentiment que, dans ce domaine précis, quelque chose avait changé en Russie en l'espace d'une décennie, ou bien c'était le milieu local qui était plus ouvert et cosmopolite.

\section{Séjour de terrain}

6 Nous sommes arrivés à Petrozavodsk le 22 avril au matin par le train de nuit parti la veille de la Gare de Ladoga à Saint-Pétersbourg, émerveillés encore par cette ancienne capitale bâtie sur le delta de la Neva par Pierre le Grand et qui n'a rien à envier aux plus belles villes du continent. En Carélie, des lacs et des forêts vantés par les guides touristiques, nous n'avons vu par la fenêtre du train que des plans d'eau encore couverts de glace et des kilomètres d'arbres, pas toujours au mieux de leur forme. La capitale carélienne nous est apparue comme une ville dynamique, ouverte, à fort potentiel, mais qui a souffert de la transition économique non aboutie: des immeubles délabrés jouxtent des centres commerciaux modernes, des trolleybus d'un autre âge se partagent la route avec des voitures de luxe, la pauvreté côtoie la richesse la plus extravertie. Mais, sauf à la gare lorsqu'on voit partir des trains pour Murmansk, au bord de la mer de Barents finalement assez proche, on n'a jamais l'impression d'être " au bout du monde »: le service hôtelier, bancaire, les possibilités de restauration, de connexion Internet, sont tout à fait satisfaisantes et garantissent au chercheur un séjour convenable, propice au travail, du moins dans la capitale de la République.

Loin d'être aussi bien ficelé que le programme de nos précédentes missions en Russie ce qui était loin de nous déplaire, sensibles que nous étions à la liberté d'agir et de décider comment et avec qui travailler sur place $^{12}-$, le programme de cette mission était très riche. La collaboratrice scientifique de l'Institut de langue, littérature et histoire de Petrozavodsk, S. Kovaleva, était notre principal contact sur place ${ }^{13}$, et c'est notamment grâce à elle que nous avons pu rencontrer les principaux acteurs de la revitalisation du vepse. En effet, l'exemple vepse est intéressant en ce qu'une véritable entreprise de "sauvegarde" de la langue menacée s'est mise en place à partir des années 1990, qui a peu d'équivalents ailleurs : universitaires, chercheurs, travailleurs 
du domaine culturel, instituteurs, tous locuteurs de cette langue en voie de disparition, ont entrepris de la revitaliser, de lui donner un nouveau souffle, de la faire (re)vivre aussi bien en ville qu'à la campagne et mènent, encore aujourd'hui, un véritable combat contre le temps et l'inexorable assimilation des Vepses, travail qui a de quoi susciter de l'admiration ${ }^{14}$. Notre projet d'enquêtes sur place a donc naturellement été orienté vers ces « activistes $»^{15}$ de la langue minoritaire.

8 Nous avions prévu deux types de questionnaires : un questionnaire sociolinguistique, centré sur l'aménagement linguistique "de par en bas » ou "à mi-palier », mené précisément par les universitaires, les associations et les organisations de développement culturel que nous venons de mentionner ${ }^{16}$, et un questionnaire phonologique. Nous avions aussi prévu de consulter le fonds documentaire existant. En ce qui concerne ce dernier, nous avons effectivement eu l'occasion de consulter l'essentiel de ce qui a été publié sur le vepse, et en vepse, ces dernières années, surtout à Petrozavodsk ${ }^{17}$. Il faut dire également que, à la différence de nos deux précédents séjours en Russie, on trouve aujourd'hui un certain nombre de ces publications assez facilement en version PDF sur Internet ${ }^{18}$. D'autres documents nous ont été offerts par les différents collègues sur place, ou nous en avons fait l'emplette auprès de la maison d'édition « Periodika $»^{19}$.

C'est précisément avec la directrice de cette maison d'édition, Lidija Rämenen, que nous avons débuté nos entretiens sociolinguistiques, ainsi qu'avec le journaliste du mensuel vepse Kodima et poète Nikolaj Abramov, les rédactions de différents journaux en langues minoritaires se trouvant précisément dans le bâtiment de la maison d'édition «Periodika ». C'est avec N. Abramov que nous avons réalisé également nos premiers enregistrements en vepse central, dans la mesure où il est originaire de Ladva (oblast de Leningrad). Nos principaux interlocuteurs au Centre scientifique carélien ont été Zinaida Strogal'ščikova, ethnologue et historienne, Nina Zajceva et Ol'ga Žukova, linguistes. Les deux dernières ont accepté de traduire systématiquement en vepse leurs réponses données en russe à nos questions. Enfin, grâce aux contacts fournis sur place par notre collaboratrice principale, nous avons eu l'occasion de visiter également les locaux de la télévision et de la radio et de nous entretenir avec le responsable du programme en langues minoritaires, Aleksandr Eremeev (en russe, finnois et livvi), et avec les journalistes de la rédaction vepse (télévision et radio), Marija Filatova et Larisa Smolina, en russe et vepse simultanément. Le dernier entretien à Petrozavodsk, avant le départ pour la campagne de la zone vepse nord ${ }^{20}$, a eu lieu au Musée des cultures nationales, avec la jeune chargée de communication, Anna Anhimova. Cette première série d'entretiens totalise 4,5 heures d'enregistrement. Exceptés les deux responsables de la maison d'édition et du programme en langues minoritaires, qui ne sont pas locuteurs du vepse et avec lesquels nous nous sommes surtout entretenus de la politique éditoriale ou d'information concernant les langues minoritaires de Carélie de façon générale, tous les autres sont locuteurs de la langue. L'essentiel de l'entretien portait précisément sur leur action au quotidien pour assurer une survie à la langue vepse. Celle-ci passe par une intense activité de publications scientifiques pour les chercheurs de l'Institut de langue, culture et histoire, par un travail journalistique couvrant les principaux supports médiatiques (presse, radio et télévision), la valorisation de la langue à travers de nouvelles technologies (programmes multimédias, collecte et mise en ligne de corpus oraux et écrits) ou encore l'activité littéraire. À chaque fois, les « activistes » soulignaient leur attachement profond à cette langue, souvent langue d'héritage familial à défaut d'être leur langue maternelle. Ils 
décrivaient les résultats obtenus dans leurs domaines respectifs, se disaient optimistes quant à l'avenir de la langue, tout en étant conscients des limites de leurs actions à long terme, face à l'assimilation galopante en faveur du russe, langue de grande fonctionnalitée ${ }^{21}$.

En effet, ces premiers jours passés dans la capitale carélienne nous ont permis de constater à quel point les langues minoritaires y manquaient de visibilité. Dépourvus de statut officiel, le vepse, le carélien, le livvi et le lude, de même que le finnois, sont invisibles et surtout inaudibles au quotidien à Petrozavodsk, reculant toujours plus sous la pression du russe, malgré une certaine reconnaissance patrimoniale pour trois d'entre elles : finnois, carélien et vepse. Pratiquement aucun panneau de signalisation bilingue n'est visible à la ronde. L'administration fonctionne entièrement en russe, seule langue qu'on entend d'ailleurs dans les rues de la capitale de la république de Carélie, qui semble coupée de tout environnement finno-ougrien. Petrozavodsk est donc une ville russe dans tous les sens du terme. Un séjour à la campagne devait nous permettre d'observer plus ou moins les mêmes conditions de défection diglossique en milieu rural ${ }^{22}$.

11 En effet, nous avons souhaité durant notre séjour rencontrer également des locuteurs souvent présentés comme les derniers locuteurs -, difficilement accessibles dans la capitale, et dont nous avons entendu parler par les différents « activistes », qui, bien évidemment, les connaissent personnellement, travaillent avec eux en se nourrissant et se ressourçant régulièrement de ce terreau que représente la campagne. Il est tout à fait significatif que, malgré la distance et les transports en commun pas toujours très fréquents ni commodes, les contacts entre la ville et la campagne sont constants : les " activistes " parcourent la campagne à la recherche de la parole vivante, les locuteurs sont souvent invités en ville pour participer aux émissions à la télévision, aux concours organisés par les chercheurs, aux différentes manifestations culturelles. Les stages et les formations sont fréquemment organisés et les différents acteurs et protagonistes du « réveil » vepse y sont souvent invités. Ces invitations ont parfois changé le cours de leur existence, tel ce participant vepse d'un stage de formation pour maîtres d'école organisé à Šoltozero en 1991 qui, en découvrant la traduction en vepse d'un texte religieux, éclata en sanglots, ému d'entendre sa langue maternelle, si mal en point, jouer un rôle aussi prestigieux à ses yeux ${ }^{23}$.

12 Notre choix de la zone vepse nord, l'une des trois zones de peuplement des Vepses ${ }^{24}$, était dicté par des raisons pratiques - une relative proximité géographique, mais surtout le fait que les efforts de revitalisation du vepse se laissent observer essentiellement dans cette zone-là à l'époque contemporaine, à la différence des années 1930 où le centre névralgique de la standardisation du vepse était surtout la région centrale. Comme nous avons été logés dans le village de Drugaja Reka, nous avons parcouru une bonne partie de cette micro-région à la recherche de locuteurs. Un détail nous a frappés d'emblée : alors qu'en Mordovie nous avons été souvent accompagnés lors de notre séjour à la campagne, astreints à la visite protocolaire auprès de l'administration locale, ici nos collaborateurs locaux nous ont simplement déposés chez nos hôtes et sont repartis en ville aussitôt pour ne revenir que le lendemain. Les différentes visites se sont faites au gré des rencontres fortuites, par exemple avec les voisins de notre logeuse, ou encore elles ont été organisées depuis la capitale, comme celle de l'école primaire à Rybreka. En ce qui concerne l'aspect général de la campagne vepse, là aussi, elle nous a semblé plus riche que ce qui nous a été donné à voir en 
Mordovie. Les routes sont davantage asphaltées, les maisons, même si souvent en bois, semblent en meilleur état, avec parfois tout le confort. L'alcool semble faire moins de ravages qu'ailleurs en Russie. Nous supposons que cela est dû au chômage moins élevé et aux possibilités offertes d'une part par l'industrie du bois, d'autre part par le travail dans des carrières. Apprenant que nous étions Français, nos différents interlocuteurs s'empressaient de nous dire que le sarcophage de Napoléon fut fabriqué précisément en grès de Carélie (quartzite de Šokša). Cette information semblait être détenue aussi bien par les paysans et les ouvriers de la campagne que par les universitaires. Les gens travaillent et semblent vivre, si ce n'est pas toujours confortablement, du moins décemment, de leur travail. Le fait que les villages de la zone vepse nord se trouvent à proximité du lac Onega constitue également un atout. Même s'il ne nous a pas été donné de visiter ces villages en été, nous imaginions aisément l'aspect pittoresque latent de ces rives du lac Onega qui, début mai, était encore couvert de glace. Quant aux discussions que nous avons pu avoir avec les habitants, lorsqu'elles ne concernaient pas l'usage et les pratiques de la langue vepse, elles tournaient souvent autour de la situation économique : prix des terrains, permis de construire, exode rural des jeunes vers Petrozavodsk ou vers la Finlande voisine; rarement autour de la situation politique, si ce n'est pour mentionner l'existence des îles Solovki à proximité (oblast d'Arhangel'sk), et encore davantage dans un sens touristique et pour évoquer une période trouble de l'histoire de l'URSS - cet archipel, qui abrite le magnifique monastère Solovetski du $\mathrm{Xv}^{\mathrm{e}}$ siècle, fut converti en camp de travail au début de l'ère soviétique et servit de laboratoire pour ce qui allait plus tard devenir le Goulag. Lorsque les hommes politiques russes ont été mentionnés, c'était toujours par rapport à la Carélie: B. El'tsin aimait venir pêcher sur les rives du lac Onega, V. Putin serait d'origine vepse, nous a-t-on même dit au cours d'un repas... Nous avons également pu constater que les habitants de Carélie, toutes nationalités confondues, étaient très fiers de leur région : combien de fois nous avons dû nous justifier pour avoir décliné la visite organisée de l'île de Kiži. Non que la visite de ce joyau architectural, avec ses églises en bois vieilles de plusieurs siècles, nous aurait déplu, mais nos salaires modestes d'enseignants-chercheurs français ne nous permettaient pas de nous payer ce voyage $\mathrm{ni}$ en hélicoptère, ni en motoneige sur la glace fragile d'un mois de mai, et surtout, nous obligeaient à concentrer nos moyens sur l'objet même de notre séjour de terrain - la langue vepse et les lieux où elle est parlée, peut-être plus pour longtemps. Cette fierté transparaît également à travers leurs dires sur la culture vepse: que penser de l'élection de Miss vepse, impensable il y a à peine deux décennies? Plus qu'un concours somme toute banal, c'est l'histoire d'une dignité retrouvée qu'on peut y voir. Un long chemin a été parcouru depuis l'histoire de cette jeune fille de la campagne qui se serait suicidée lorsque son identité vepse avait été découverte en ville ${ }^{25}$ : à notre époque, on exhibe fièrement ses origines et sa culture.

Durant notre séjour à la campagne, nous avons visité et réalisé des entretiens dans quatre villages : Drugaja Reka, Rybreka, Šeltozero et Šokša. Les onze informateurs, pour la plupart retraités, excepté les institutrices des écoles maternelle et primaire de Šeltozero et Rybreka, ont tous répondu à nos questions en russe et en vepse, certains avec moins de facilité que d'autres, ne comprenant pas toujours l'intérêt de répéter en vepse ce qui avait déjà été dit et ce que nous pouvions très bien comprendre en russe. Les enregistrements, tous filmés, totalisent 6,5 heures environ. Certains se sont prêtés au jeu d'autant plus facilement qu'ils sont habitués à côtoyer des chercheurs - telle par exemple V. Mironova de Rybreka : elle a déjà eu l'occasion de participer aux concours 
linguistiques à Petrozavodsk, seule ou avec son petit-fils, également locuteur - l'un des rares parmi les jeunes, et notre collègue italienne L. Siragusa, qui nous a précédés sur le terrain, l'a déjà interviewée, tout comme les chercheurs de l'Institut de langue, littérature et histoire de Carélie. Le village de Rybreka est l'un des rares à offrir encore des cours de vepse. Nous avons eu l'occasion de filmer un cours donné par une jeune enseignante, M. Mironova, formée à l'Université de Petrozavodsk et revenue à la campagne pour y enseigner la langue, et nous avons pu constater les problèmes posés par les écarts entre le vepse standard, conçu comme une synthèse fonctionnelle de trois dialectes, et la variété dialectale de la zone nord. Il arrive que les élèves soient parfois déconcertés par les différences de vocabulaire entre la langue parlée à l'école et la langue de la maison, essentiellement celle des grands-parents, car avec les parents ils ne parlent qu'en russe. Deux mots faisaient l'objet du cours auquel nous avons assisté : löts « grenouille » et öläpak « chauve-souris ». Il s'agissait d'écrire le mot sous un dessin à colorier. La base de données lexicales en ligne du Kotimaisten Kielten Keskus de l'Université de Helsinki ${ }^{26}$ confirme en effet le lexème löts "grenouille", mais aucunement öläpak pour " chauve-souris ", qui est apparemment un calque du finnois yölepakko habillé phonologiquement en vepse (le dictionnaire du vepse-russe donne la forme öläpäkaine, Zajceva, 2010 : 491). En effet, la base de données recense les formes suivantes, dont la phonologie diffère de la forme utilisée dans le cours, et confirme la dérivation diminutive, absente dans öläpak :

\begin{tabular}{|c|c|c|}
\hline Vepse & Flexion & Finnois \\
\hline elapakeinine & -žęd & yölepakko \\
\hline üötilakck & -on, -oid, -oiden & yölepakko \\
\hline ölapakeiné & & yölepakko \\
\hline öpärränée & -räšst, -rāžid, -răzzęd & yölepakko \\
\hline
\end{tabular}

On comprend que l'institutrice ait utilisé ce calque: les formes ci-dessus sont particulièrement complexes, et toutes dérivées à l'aide de diminutifs. Mais le problème reste le même: lorsque les enfants, enthousiastes, répètent la forme apprise à l'école avec la maîtresse auprès de leurs grands-parents, personne ne les comprend ${ }^{28}$. De tels décalages sont propices à installer un scepticisme durable sur l'efficacité ou la légitimité de l'enseignement de la langue minoritaire. De toute évidence, le choix de ces deux mots était conditionné par le fait que l'institutrice avait sous la main des images et des coloriages représentant une grenouille et une chauve-souris (cf. en annexes le cliché « Un cours de vepse. École primaire de Rybreka »). Le choix de la grenouille était certes judicieux mais le choix de la chauve-souris n'était pas, à notre sens, une option réaliste pour entreprendre l'apprentissage d'un mot nouveau en vepse pour des enfants d'école primaire. D'autres mots plus simples dans leur forme phonologique et immédiatement intelligibles pour les parents ou grands-parents des élèves auraient été préférables à ölepak, calque opaque pour un locuteur de vepse dialectal. Par exemple, puisque la lettre de l'alphabet qui faisait l'objet de la leçon était apparemment la dernière lettre de l'alphabet < 0 >, un mot composé comme öpu « perchoir » (équivalent 
finnois yöpuu : la perche sur laquelle les poules se mettent pour passer la nuit) aurait été préférable.

En effet, les grands-parents ne comprennent pas toujours l'intérêt d'un standard et refusent d'admettre qu'il s'agit bien d'une seule et même langue: si le vepse aujourd'hui s'écrit en alphabet latin - admettons que cela puisse être un frein pour des personnes âgées habituées au cyrillique -, c'est bien la même langue que celle qu'ils parlent, ou qu'ils ont toujours parlée. D'autres semblent même complètement réfractaires à ce vepse standard créé dans les années 1990 à partir des formes de l'oblast de Leningrad: telle cette locutrice qui voulait généreusement nous offrir un livre «en finnois » - et pas n'importe lequel : la Bible -, sans se rendre compte qu'il s'agissait d'un livre en vepse, sa langue maternelle. Le fait que celui-ci soit écrit en alphabet latin a suffi pour qu'elle n'ait ni l'envie ni la curiosité de l'ouvrir et qu'elle le catégorise comme un livre en langue étrangère.

Nous fûmes frappés par la diversité des caractères et des attitudes des locuteurs que nous avons rencontrés sur le terrain, dans les petits centres urbains et à la campagne, dans la région vepse nord. Par rapport à nombre de situations que nous avons connues dans le monde (ailleurs en Russie, Pays baltes, Pays basque, Italie, Balkans, domaine d'oïl, Amérique centrale, etc.), le terrain vepse avait quelque chose de particulier: chaque locuteur nous a semblé suivre son chemin comme circonscrit dans un monde à lui, avec une idée très claire de ce dont il voulait parler ou qu'il acceptait de faire avec nous pour nous accompagner dans notre approche du vepse. La propension au monologue était souvent frappante, probablement en raison du caractère artificiel de l'enquête, même lorsqu'elle se présente sous forme d'un entretien semi-directif, sur des questions relatives au temps passé aussi bien qu'à leurs représentations de leurs propres pratiques langagières aujourd'hui. Probablement aussi en raison de nos propres limites et compétences, en tant qu'enquêteurs peu familiers de ce terrain. Initialement, nous avons obtenu des récits de vie en vepse à partir de questions posées en russe, mais cette méthode par défaut renforçait la tendance au monologue - nous avions compté sur l'aide d'enquêteurs vepsophones issus des institutions universitaires censés nous accompagner, mais cette option se révéla finalement impossible, faute de disponibilité des chercheurs pour nous rejoindre sur le terrain. Nous avons donc adopté une technique simple que nous avions observée dans les enregistrements réalisés dans la seconde moitié du siècle dernier chez certains chercheurs finlandais: Jean Léo Léonard posait les questions en vepse, quitte à surmonter le sentiment d'insécurité linguistique dans l'utilisation d'une langue rêvée depuis trente ans, étudiée de manière livresque, mais jamais pratiquée. La connaissance livresque du vepse et ses compétences en finnois et en estonien lui rendaient la langue transparente : peu de choses lui échappaient dans les récits de vie que nous écoutions.

Cette sensation de tout comprendre était pour lui une découverte inattendue. Elle était renforcée par la nécessité : l'enquête exigeait un effort constant de communication en vepse de la part d'un des deux enquêteurs, aussi bien pour écouter et comprendre que pour parler et tenter, par des tactiques ad hoc d'apprenant de langue étrangère, de se faire comprendre - l'expérience de Laura Siragusa est très semblable, d'après ce qu'elle nous en a dit lors d'un entretien sur son expérience d'enquêtrice en terres vepses. Mais nous étions souvent confrontés à la volonté inébranlable de nos interlocuteurs : telle enquête sur les proverbes par exemple échoua lamentablement, car le thème n'intéressait pas notre informatrice - ni son mari, qui suivait de loin l'interaction en 
exprimant son scepticisme « on ne dit plus toutes ces vieilleries, c'est fini, tout ça, ça ne sert à rien... ». Telle autre informatrice ne voulait parler que de toponymie car elle avait été classée à un concours sur ce sujet, si bien qu'elle évacuait tout autre thème de conversation. Tel informateur n'avait guère envie de répondre à un questionnaire phonologique pourtant simple, en répétant trois fois les formes en vepse, selon la technique courante d'élicitation. Un couple âgé de 70 ans refusait de parler vepse ou n'acceptait de le faire qu'en réalisant l'une des prédictions que nous avions entendues avant notre départ ( ils parleront, mais en vepse mélangé de russe, et ce sera inextricable»). Telle autre locutrice, à peine sollicitée sur les questions relatives à la société vepse qu'elle avait connue durant son enfance, ne cessait plus de parler, tantôt entièrement en russe, tantôt entièrement en vepse, pour de longues périodes impossibles à interrompre. Certains encensaient le passé, représenté comme un âge d'or, d'autres ne voulaient ni en parler ni en entendre parler. Plus qu'ailleurs, nous sentions que c'était au chercheur de s'adapter coûte que coûte à son interlocuteur, sans compter sur plus de plasticité. Il fallait donc à chaque fois trouver le bon angle d'approche. Aucun questionnaire préétabli ne semblait susceptible de vraiment fonctionner. La mémoire collective semblait fragmentée en autant de visions parcellaires qu'il y avait de locuteurs, à la différence de tant de terrains que nous avons connus, où un consensus et un corpus commun émergent rapidement et massivement au sein d'une société dialectophone rurale, intarissable sur les techniques agricoles, les cycles calendaires, les cycles festifs et les rites de passage. Nous avions parfois l'impression d'être entrés dans le monde d'Alice au pays des merveilles, où l'art du coqà-l'âne est le principal mode opératoire.

Nous fûmes encore plus stupéfaits lorsqu'une voisine fit littéralement irruption au cours d'un entretien avec une ancienne institutrice, intensément engagée dans la narration des cycles festifs dans un vepse d'une qualité éblouissante, pour se lancer à son tour dans un récit parallèle, couvrant la voix de celle qui était engagée dans son récit. Cette polyphonie n'était pas momentanée ou due à un raté d'ajustement interactionnel : elle dura tout au long de l'entretien amorcé avec sa voisine, sans que cette dernière en prenne ombrage. Cela ne retirait rien à la sympathie de ces personnes, dont l'attitude était tout à fait cordiale, mais l'impression de voyage en terres d'Alice devenait de plus en plus forte, d'une enquête à l'autre. Les cadres classiques de l'interaction nous semblaient souvent pulvérisés, sans que nous puissions trouver une autre explication que, sans doute, notre impréparation. C'est d'ailleurs ce qui nous motive ici à relater ces anecdotes, car elles pourraient s'avérer utiles aux chercheurs pour des séjours de terrain futurs. Notre conseil est alors : vous qui entrez en terres vepses, oubliez tout protocole d'enquête préétabli, laissez-vous guider par les individualités, ne vous obstinez pas à imposer un thème ou un mode opératoire. Mais ne soyez pas non plus timides, ni effacés, ni indécis. Sachez trouver l'angle d'approche le plus idiosyncrasique, le plus adapté à la personnalité de votre interlocuteur. Ne vous offusquez pas des polyphonies et des conquêtes de l'espace conversationnel ou narratif par des tiers. Laissez-vous porter, mais pas emporter - et ne vous emportez jamais ni d'aucune façon, cela va de soi. Parlez vepse. Même si vous pensez ne pas le parler, pourvu que vous ayez une bonne connaissance préalable de cette langue - en outre, rares sont les chercheurs finno-ougristes se rendant en terrain fennique qui ne maîtrisent pas le finnois ou l'estonien, ce qui permet une compétence au moins passive très enviable, si on compare à la détresse du finno-ougriste fennisant ou magyarisant se 
trouvant soudainement plongé en immersion dans le mordve ou le komi sans une connaissance approfondie de ces langues.

Quoiqu'il en soit, ces cadres interactionnels quelque peu décalés ont l'avantage de briser les routines et de pousser le chercheur à aller au-delà de ses limites. Il est arrivé que nos interlocuteurs nous félicitent pour quelques phrases en vepse donnant l'illusion que nous le parlions, tout comme il est arrivé que la réaction de l'informateur soit négative ou disqualifiante :

Pourriez-vous plutôt poser votre question en russe? Je ne comprends rien à votre

vepse,

Bon, on voit que vous ne le parlez pas vraiment....

Là encore, c'est de bonne guerre - et c'est même réaliste.

De retour à Petrozavodsk, c'est sur le monde des écoles que nous nous sommes concentrés, en filmant deux cours à l'université et en interviewant l'enseignante de vepse de l'école finno-ougrienne de la capitale, N. Kukoeva (1,5 heure environ). Dans toutes les écoles, les enseignants nous ont fait part de ce phénomène que l'on observe dans de nombreuses situations minoritaires: dissolution progressive des infrastructures existantes, puisque nombre de parents préfèrent inscrire leurs enfants dans des classes monolingues russes pour augmenter les chances de mobilité sociale de leurs enfants, alors que des conditions existent souvent pour un enseignement de qualité dans des langues autres que le russe. Et pourtant ce n'est pas le moindre des paradoxes que de constater que, dans ce cas précis, de nombreux jeunes ont pu intégrer une université ou trouver du travail en Finlande, précisément grâce à leur maîtrise non pas $d u$ russe, certes valorisante, mais du vepse, ou du carélien. Cet exode volontaire vers la Finlande nous a laissé un goût amer. Nous étions tentés de comprendre, ou de partager, la joie des parents, souvent d'ailleurs activistes eux-mêmes, nous racontant les succès universitaires et professionnels de leurs enfants à Helsinki ou à Tampere, leur facilité d'adaptation grâce à une scolarité dans des cursus finno-ougriens, et le sentiment de gâchis : si les jeunes locuteurs potentiels du vepse, des personnes déjà sensibilisées à la langue et la culture, quittent la Carélie, qui pourra y assurer un avenir pour les langues minoritaires?

21 Enfin, l'une de nos plus grandes découvertes, de retour à Petrozavodsk, a été le club de discussion vepse: Paginklub. Coordonné par la jeune journaliste de la radio Larisa Smolina qui assure également la gestion de la page Internet correspondante ${ }^{29}$, la participation au club de discussion le 29 avril $2013^{30}$ nous a permis d'observer comment se met en place et se structure une communauté de pratiques (cf. Eckert \& Wenger, 2005). Des locuteurs de la langue, quels que soient leur niveau, leurs compétences à l'oral et à l'écrit ou leurs liens avec la langue vepse, participent à ces rencontres et s'activent pour certains sur la page Internet, donnant à cette langue une visibilité insoupçonnée. On est cette fois dans une zone de confort, une niche sécurisée pour l'apprenant, qui ne risque pas de se voir disqualifié par des locuteurs sûrs d'eux et au franc parler, ou qui peut se contenter d'écouter simplement, s'il n'ose parler. On accède au vepse "à son rythme ", dans une ambiance conviviale, où on échappe aux jugements - en tout cas, aux jugements frontaux.

Premièrement, nous avons découvert - même si l'on pouvait s'en douter - que toutes les personnes impliquées dans la revitalisation de la langue aujourd'hui se connaissent, se fréquentent et travaillent ensemble, formant un véritable réseau qui couvre tous les cercles où peut se jouer l'avenir de la langue (recherche, enseignement, médias...). Mais 
autour de ces mêmes personnes gravitent d'autres locuteurs, aussi bien de la ville que de la campagne, qu'elles font participer aux différentes activités organisées. Deuxièmement, si l'on regarde attentivement la page Internet du Paginklub, on constate que les différentes personnes que nous avons rencontrées à la campagne ne sont souvent pas coupées de « la ville » et des activités qui s'y déroulent, comme on pourrait le penser lorsqu'on réfléchit à leurs conditions de vie dans des villages éloignés de plusieurs dizaines de kilomètres de la capitale. Ainsi, on peut voir le même M. Pidžakov, locuteur vepse qui nous a reçus chez lui dans le village Šokša, sur les plateaux de la télévision locale, ou encore dans le film documentaire «La vie de la maison vepse » de L. Smolina et V. Slavov, présenté au « Festival du film écologique Barents » auquel nous avons participé le 25 avril 2013 à Petrozavodsk. Cette impression de rencontrer souvent les mêmes personnes partout où il est question des Vepses a quelque chose de rassurant pour les chercheurs, mais revêt également un aspect inquiétant: s'il est, en effet, rassurant de constater cette synergie entre les mondes académique et urbain et celui de la campagne où chaque locuteur compte et chaque locuteur est valorisé, il est en revanche inquiétant de se rendre compte très vite que l'on tourne dans un cercle relativement fermé où de nouveaux locuteurs sont rares, et où les jours de la langue sont comptés.

\section{Conclusion : horizons et microcosmes}

Du point de vue quantitatif, les objectifs de cette mission ont été atteints, en dépit de la durée limitée de notre séjour: nous avons pu recueillir une douzaine d'heures d'enregistrements de très bonne qualité sonore, presque entièrement filmés, avec des contenus aussi divers que des entretiens sociolinguistiques en russe, finnois ou vepse, des conversations spontanées dans des clubs de discussion carélien et vepse, des lectures de textes (poèmes), des récits de vie en vepse, des morceaux de chants traditionnels.

Dès notre retour en France, nous avons commencé à exploiter les matériaux, à travers les communications orales et les articles soumis ou déjà publiés ${ }^{31}$. À la réflexion, il nous est même apparu que, malgré les réserves ou les craintes que nous avons exprimées plus haut au sujet d'une certaine circularité de l'aménagement linguistique du vepse par un réseau limité d'activistes, nombre d'éléments de la revitalisation vepse sont en quelque sorte exemplaires. Nous nous risquons en effet à penser que, si la situation démographique de la langue était moins grave (à savoir, la majorité de la population vepsophone est âgée de plus de 50 ou 60 ans, principalement dans les campagnes) et si l'appui institutionnel, notamment dans l'éducation primaire et secondaire, était davantage volontariste, le «modèle de revitalisation vepse " serait un parangon de ce qui peut ou doit être fait pour juguler ou renverser la tendance à l'assimilation d'une langue minoritaire en danger. Parmi les points forts, on peut mentionner la complexité et l'intrication du dispositif, ainsi que les interactions entre l'aménagement « de par en bas ", «à mi-palier » et "de par en haut». Complexité et intrication car ce sont de multiples agents qui œuvrent ici à la valorisation du vepse, tous engagés dans des interactions de haute densité : le circuit Radio-Télévision \& reportages / Université / centres de recherches / Paginklub / revues \& publications, se rétroalimente en permanence, et ne cesse de produire documents et opportunités. Les activités et les initiatives sont enchevêtrées et se soutiennent ou se renforcent mutuellement, et elles 
se ressourcent continuellement auprès des locuteurs de la «base ». Certaines initiatives, comme les concours de toponymie, qui pourraient paraître quelque peu décalées par rapport aux préoccupations quotidiennes des populations, remportent en fait un franc succès. Une sorte de mobilisation permanente travaille le terrain, évitant de cantonner l'action seulement au milieu urbain. C'est dans ce va et vient permanent entre la « base » (les locuteurs des villages, donc le palier « de par en bas ») et le niveau "à mi-palier» (à savoir, les centres de recherche, l'Université, un établissement d'enseignement secondaire comme l'école finno-ougrienne) qu'il faut rechercher l'intrication de ce système complexe. Par ailleurs, ces deux sphères de travail sur et pour la langue ne seraient guères viables si elles n'étaient appuyées, "de par en haut ", par une politique bienveillante du gouvernement de la république de Carélie. Or, là encore, ce palier élevé est alimenté par les deux précédents, « de par en bas » et « à mipalier », puisque nombre de fonctionnaires ou d'élus des services culturels de la république de Carélie ou bien sont issus des villages, ou bien ont été formés à l'école finno-ougrienne, telle cette responsable rencontrée dans les bureaux du gouvernement carélien et originaire d'un village de la région vepse nord, qui s'exprimait dans un finnois et un vepse parfaits et avait été sensibilisée aux questions fenniques par sa scolarité. Tel autre cadre de la radio-télévision de Petrozavodsk était d'origine olonetsienne, et parlait couramment finnois et livvi - nous eûmes le plaisir de constater sa capacité dans ces deux langues au cours de notre entretien. Autrement dit, le système éducatif à mi-palier, dans sa composante pluraliste intégrant les langues fenniques, même s'il reste limité à un seul établissement secondaire, s'avère un vecteur efficace de l'aménagement linguistique local. Les élites connaissent des conditions de reproduction sociale qui tiennent compte des langues fenniques locales et les valorisent dans une certaine mesure, même si ce segment reste très limité - on ne pourrait guère en dire autant en France ou même en Italie. Certes, le finnois standard reste le grand attracteur, en tant que langue de l'UE, langue scandinave, autrement dit, en tant que ressource cosmopolite et ouverture vers l'Ouest - une ouverture de proximité, éminemment stratégique. Par ailleurs, à mi-palier encore, la qualité du travail d'élaboration du corpus de la langue par les linguistes, l'infatigable production de manuels scolaires, de travaux de recherche et de vulgarisation (dont certains, même, en vepse), sont également un atout. On peut même dire que le vepse dispose d'ores et déjà d'outils lexicographiques et normatifs ou ergonomiques (grammaires, manuels de conversation, etc.) plus systématiques et efficaces que nombre de langues minoritaires en Europe, grâce à cet effort constant d'élaboration d'outils didactiques et pratiques de qualité. Paradoxalement, cette normativisation (codification et standardisation) se fait dans un alphabet peu pratiqué par les locuteurs de la base - cependant, le même handicap, pour des locuteurs âgés, peut s'avérer être un atout pour des locuteurs jeunes, orientés vers les langues étrangères occidentales. Il était frappant de voir que dans la même salle où était enseigné le vepse, on trouvait, côte à côte, des posters décrivant l'alphabet latin aussi bien pour le vepse que pour l'anglais.

Beaucoup reste à faire, toutefois, pour que ce " modèle de la revitalisation vepse » ait vraiment un impact permettant de juguler le processus d'assimilation, déjà très avancé : l'enseignement du vepse à l'école primaire tel que nous l'avons vu, avec un nombre d'heures aussi limité et des techniques pédagogiques comme celles que nous avons observées, ne peut guère changer quoi que ce soit à la tendance assimilationniste. Il manque un plan stratégique pour un véritable aménagement linguistique, alors que partout où une politique de revitalisation ambitieuse et 
volontariste a été entreprise (Pays de Galles, Communauté Autonome Basque, Généralité de Catalogne, etc.), un tel plan était le pilier de la revitalisation ou de la "normalisation sociolinguistique ». Malgré ses qualités, l'indéniable bonne volonté et compétence hors-pair de ses protagonistes, l'aménagement linguistique vepse « de par en bas » et « à mi-palier » n'est jamais, à vrai dire, qu'un cautère sur une jambe de bois, pour dire les choses très franchement. Mais la «faute » n'en incombe nullement aux activistes et aux universitaires, qui ont fait ou font tout ce qui est en leur pouvoir. Il nous semble manifeste que c'est aussi «de par en haut " que devrait se renforcer un plan d'ensemble de défense et de promotion du multilingusime fenno-russe, incluant toutes les langues fenniques de Carélie, dans une logique d'échanges transfrontaliers. Tant que l'échelle d'intervention et de réflexion sur les ressources, les méthodes, les objectifs et les supports d'un tel aménagement ne changera pas, les aménageurs seront condamnés à poursuivre sans relâche une action certes méritoire, mais qui n'en reste pas moins une forme de bricolage à échelle locale, par imbrication de projets, de réalisations médiatiques ou éditoriales, sans réelle incidence sur les pratiques langagières, et le vepse continuera à disparaître de la société locale, inexorablement.

Quoiqu'il en soit, ce terrain vepse nous a permis de constater que cette langue quasiment légendaire, depuis sa "découverte» par Elias Lönnrot, est encore une langue accessible et non dénuée d'une certaine vitalité, même si elle semble vivre "ses derniers jours" (mais pas encore ses dernières heures). Il nous a permis également d'observer un modèle en miniature d'aménagement linguistique complexe et interactif avec le milieu des locuteurs, non dénué d'une relative exemplarité. Il nous a ouvert les portes d'un microcosme riche en productions, en réalisations, en création littéraire et linguistique. Si ce petit monde rappelle parfois Alice au pays des merveilles, c'est davantage d'une manière stimulante qui pousse le chercheur dans ses retranchements, le sort de sa zone de confort et le fait progresser dans sa connaissance des sociétés humaines. Un terrain attachant, émouvant, rempli d'enthousiasme et de créativité. Un des terrains les plus passionnants que réservent encore les langues ouraliennes de Russie, à deux pas de l'Union européenne, dans une région méconnue, d'une grande diversité. De la légende (le fabuleux destin de la langue tchoude, inauguré par Elias Lönnrot) à la réalité (une langue en danger, hautement documentée et intensément soutenue par un activisme de bon aloi et compétent), le vepse apparaît comme une langue-monde en miniature, surtout si l'on regarde au-delà du seul horizon de l'arrière-pays de Petrozavodsk et de la Carélie orientale, pour explorer les régions plus au sud, dans l'oblast de Leningrad et ses alentours - région plus marginale, où l'exode rural a, paraît-il, désertifié les villages - ou si l'on s'intéresse également à la zone lude, qui est pour nous une variété vepse de continuum avec le livvi ou carélien d'olonets (Aunus).

\section{BIBLIOGRAPHIE}

DJORDJEVIC Ksenija, 2005a, « Les revendications linguistiques des Mordves, des Maris et des Oudmourtes avant et après la Révolution d'octobre 1917 du point de vue de la standardisation », 
in ALÉN Garabato Carmen (dir.), L'éveil des nationalités et les revendications linguistiques en Europe (1830-1930), Paris, L'Harmattan, p. 89-102.

DJORDJEVIC Ksenija, 2005b, « L'aménagement linguistique en Mordovie : problèmes et perspectives », Paris, Etudes finno-ougriennes, $\mathrm{n}^{\circ} 36$, p. 45-64.

DJORDJEVIC Ksenija, 2006a, « Frontière, territoire et enclaves linguistiques et culturelles : langues trans- et intrafrontalières », Montpellier, Lengas, $n^{\circ}$ 60, p. 99-115.

DJORDJEVIC Ksenija, 2006b, « Mordve, langue minoritaire, langue minorée : du discours officiel à l'observation du terrain », Paris, ELA, $n^{\circ} 143$, « Langues minorées, langues d'enseignement ?» (coord. H. Boyer), p. 297-311.

DJORDJEVIC Ksenija, 2007, « L'enseignement du français à la périphérie de la Russie centrale : compte rendu d'une observation ", in THYRION F. \& F. FLAMINI (éds.), Variation et interculturel dans l'enseignement du FLE : Objectifs spécifiques et contextes d'apprentissage, Cortil-Wodon (Belgique), E.M.E. \& InterCommunications, p. 253-269.

DJORDJEVIC LEONARD Ksenija, 2013, « Dynamique sociolinguistique de la langue vepse », in Harri VEIVo et Julia NYIKOS (dir.), « Marges, interstices, contacts », Cahiers d'Études Hongroises et Finlandaises, $\mathrm{n}^{\circ} 19$, Paris, L'Harmattan, p. 87-99.

DJORDJEVIC LEONARD Ksenija 2014 (à paraître), « La mise en visibilité de la langue vepse », Actes du colloque Les minorités invisibles : diversité et complexité (ethno)sociolinguistiques, coord. Ksenija Djordjević Léonard, 28-29 novembre 2013, Université Montpellier 3.

DJORDJEVIC Ksenija \& LEONARD Jean Léo, 2006, Parlons mordve, Paris, L’Harmattan.

ECKERT Penelope \& WENGER Étienne, 2005, « Communities of practice in sociolinguistics », Journal of Sociolinguistics, vol. 9-4, p. 582-589.

KETTUNEN Lauri, 1922, Lõunavepsa häälik-ajalugu 1-2. Acta et commentationes Universitatis Dorpatensis B, Humaniora, Tartu, Tartu Ülikool.

KETTUNEN Lauri, 1925, Näytteitä etelävepsästä 2. - Suomi 5 :4, Helsinki, SKS.

KETTUNEN Lauri, \& SIRO Paavo, 1935, Näytteitä vepsän murteista, Helsinki, SUST 70.

KETTUNEN Lauri, 1943, Vepsän murteiden lauseopillinen tutkimus, Helsinki, SUST 86.

KETTUNEN Lauri, 1945, Tieteen matkamiehenä, 12 ensimmäistä retkeä 1907-1918, Porvoo-HKI, WSOY.

KETTUNEN Lauri, 1960, Suomen lähisukukielten luonteenomaiset piirteet, Helsinki, SUST 119.

LEONARD Jean Léo, 2007, « La variation interlangue et dialectale des langues finno-ougriennes de la Volga : planification linguistique et aspects structuraux internes », Lengas, 60, p. 115-141.

LEONARD Jean Léo, 2008a, « Simplicité de la flexion mordve ? ", Bulletin de la Société de Linguistique de Paris, Louvain, Peeters, p. 364-400.

LÉONARD Jean Léo, 2008b, « Morphs and Stems: the Realisational Drift in Uralic Morphology and the Iconicity Paradox as a Cognitive Asset for Language Evolution Theory (LET) », communication orale, International Conference on Cognitive and Functional Perspectives on Dynamic Tendencies in Languages, 29 mai-1 ${ }^{\mathrm{er}}$ juin 2008, Université de Tartu, Estonie.

LEONARD Jean Léo, 2011, « Le vepse en tant que prisme typologique : universalité, fennicité et spécificité ou de la beauté discrète des jardins japonais en morphologie flexionnelle », journées fenniques Les peuples fenniques minoritaires, Paris. 
LEONARD Jean Léo, 2012, « Le modèle PARADIGM FUNCTION MORPHOLOGY (PFM) appliqué aux diasystèmes mordve, mazatec et euskera (flexion verbale) : perspectives heuristiques et apories », communication au Centre Iker (CNRS, UMR 5478 CNRS), Bayonne, 12 avril 2012.

LEONARD Jean Léo, 2013, « Approche socio-phonétique de la fricative laryngale en vepse », Journées d'études finno-ougriennes, Université de Paris 3, 14 juin 2013, Paris, CIEH-FI Cahiers d'Etudes Hongroises et Finlandaises, 2013, n 19, Marges, Interstices, Contacts, Paris, L'Harmattan, pp. 101-123.

LEONARD Jean Léo, 2014, « Le « chaînon manquant » trouvé sur le lac Onega : les (post)néogrammairiens face au vepse (fennique oriental, ouralien) », Journée d'études de la Société de Linguistique de Paris, 18 janvier 2014, à paraître dans les Mémoires de la SLP, n 23 (en préparation).

LEONARD Jean Léo \& SAMVELIAN Polet, 2007/2008, « La Conjugaison Objective Définie Mordve (CODM) : morphologie réalisationnelle contre morphologie incrémentielle », Colloque T.E.L. Temps, espaces, langages : La Hongrie à la croisée des chemins, Cahiers d'Études Hongroises 14, p. 151-166.

LEONARD Jean Léo \& DJORDJEVIC LEONARD Ksenija, 2014 à paraître, « Propositions exploratoires pour la documentation linguistique de langues finno-ougriennes de la Fédération de Russie », Actes de la journée d'étude Langue, marge territoriale et besoin linguistique : approche comparée entre Carélie et Pays Basque, coord. Alain Viaut, Université de Bordeaux 3, 22 novembre 2012.

LEONARD Jean Léo \& JAGUENEAU Lilianne, 2013, « Disparition, apparition et réapparition des langues d'oïl : de l'invisibilisation au nouveau regard », à paraître dans le Bulletin de la Société de Linguistique de Paris, $\mathrm{n}^{\circ}$ 108/1 (sous presse).

LEONARD Jean Léo \& JAGUENEAU Lilianne, 2014, « Vitalité et revitalisation, dévernacularisation et standardisation : trois langues d'oïl (poitevin-saintongeais, gallo, morvandiau-bourguignon) », in Actes du Colloque International organisé par l'AULF et le LESCLaP-CEP Standardisation et vitalité des langues de France, Amiens, les 13 et 14 octobre 2011 (sous presse).

LÖNNROT Elias, 1853, Om det nord-tschudiska språket, réédition 2002, Kuhmo, Juminkeko.

ROoS J.P., 1985, « Elämäntapojen tyypeistä elämänkertojen valossa », in Roos, J.P. Elämäntapaa etsimässä, Helsinki, Tutkijaliiton Julkaisusarja, p. 37-49.

SIRAGUSA L., 2012, Vepsian Language: Speaking and Writing Heritage Language in Villages and Cities, thèse de doctorat, Université d'Aberdeen, Écosse.

STROGAL'ŠČIKOVA 2008 = СТРОГАЛЬЩИКОВА 3.И., ВЕПСЫ : ИСТОРИКО-ЭТНОГРАФИЧЕСКИЙ ОЧЕРК, ПЕТРОЗАВОДСК, ПЕРИОДИКА.

TUNKELO E.A., 1946, Vepsän kielen äännehistoria, Helsinki, SKS.

ZAJCEVA \& MULLONEN 1972 = зАЙЦЕВА Н.Г. \& мУлЛонЕН М.И., Slovar' vepsskogo jazyka, Moskva.

ZAICEVA Nina \& MULLONEN Marija, 1995, Vepsä-venälaine, venä-vepsläine vajehnik, Petroskoi.

ZAJCEVA 2010 = ЗАЙЦЕВА Н.Г., НОВЫЙ ВЕПССКО-РУССКИЙ СЛОВАРЬ, ПЕТРОЗАВОДСК, ПЕРИОДИКА.

ZAJCEVA \& MULLONEN 2007 = ЗАЙЦЕВА Н.Г. \& МУЛЛОНЕН М.И., НОВЫЙ РУССКО-ВЕПССКИЙ СЛОВАРЬ, ПЕТРОЗАВОДСК, ПЕРИОДИКА.

ZAJCEVA \& ŽUCOVA 2009 = ЗАЙЦЕВА Н.Г. \& ЖУКОВА О.Ю, РУССКО-ВЕПССКИЙ РАЗГОВОРНИК, ПЕТРОЗАВОДСК, ПЕРИОДИКА. 
USPENSKIJ N.P., 1913, Tshudsko-Russkij slovar', St. Petersburg.

\section{NOTES}

1. Il s'agissait pour nous d'étudier les langues mordves (erza et mokcha), aussi bien sur le plan linguistique (à travers notamment des enquêtes phonologique et morphosyntaxique) que sociolinguistique (en particulier les conditions d'aménagement linguistique dans cette république autonome de la Fédération de Russie, une décennie après l'éclatement de l'URSS).

2. Voir, par exemple, notre ouvrage Parlons mordve : une langue finno-ougrienne de Russie, publié en 2006, ainsi que nos différents articles : Léonard, 2007, 2008a, 2008b, 2011 et 2012, et Djordjević, 2005a, 2005b, 2006a, 2006b et 2007, puis Léonard \& Samvelian, 2007/2008.

3. En estonien : Keele ja Kirjanduse Instituut.

4. Mentionnons, entre autres, les travaux du célèbre folkloriste finlandais Elias Lönnrot 1853 ou du linguiste August Ahlqvist au XIX ${ }^{\mathrm{e}}$ siècle, ceux de Lauri Kettunen 1920, 1922, 1925, 1943, de Kettunen \& Siro 1935, ou d'Uspenskij 1913, Zajceva \& Mullonen 1972, 1995, Zajceva 1981, 2000, 2003 plus récemment.

5. Lors du recensement de 2010, 5936 personnes se sont déclarées comme Vepses en Russie, dont 3423 en Carélie, tandis que 3613 personnes ont déclaré parler la langue vepse.

http://www.gks.ru/

6. Nous nous sommes retrouvés à cette occasion, bien malgré nous, mêlés à un conflit interne entre les enseignants et chercheurs de l'université de Saransk et de l'Institut des Humanités, et une chercheuse de statut apparemment indépendant qui nous avait été recommandée par des collègues français insuffisamment informés.

7. Bien que notre deuxième séjour de terrain en Mordovie, en 2004 se fût nettement mieux passé que le premier.

8. La coordination en était assurée par Alain Viaut pour la partie française, et par Svetlana Moskvičeva, pour la partie russe.

9. Ils ont notamment réalisé plusieurs enquêtes (questionnaires et entretiens semidirigés) à Petrozavodsk, en 2011, auprès d'un échantillon d'acteurs institutionnels et associatifs ainsi que des locuteurs du carélien. Les enquêtes se sont poursuivies au cours des années suivantes.

10. Jean Léo Léonard tient à remercier l'Institut Universitaire de France pour le financement de sa mission en Russie. Ksenija Djordjević Léonard remercie Dipralang (EA 739, université de Montpellier 3) pour sa contribution financière.

11. Les visas portaient la spécification "collaboration scientifique et technique », avec la précision qu'il s'agissait bien d'une « mission de collecte de matériaux linguistiques pour des travaux de recherche sur la langue vepse» [экСПЕДИция ПО СБору ЛИНГВИСТИЧЕСКОГО МАТЕРИАЛА ДЛЯ НАПИСАНИЯ ИССЛЕДОВАНИЯ О ВЕПССКОМ ЯЗЫКЕ].

12. En 2003, la partie russe nous avait non pas suggéré, mais imposé un programme d'enquêtes de terrain dans une série de localités, en prévoyant des accompagnateurs, 
des nuitées d'hôtel, alors que nous ne demandions qu'à séjourner dans une ou deux fermes afin de connaître la vie de villages mordves au quotidien et de nous concentrer sur une ou deux variétés. Le refus de ce programme imposé avait eu pour effet de contrarier les instances officielles, qui nous avaient signifié par fax leur intention d'annuler notre invitation et notre visa à l'arrivée sur le territoire russe si nous persistions à préférer le programme initial, pourtant conforme à nos souhaits. Nous ne nous rendîmes compte qu'après coup que l'affaire était plus complexe qu'il ne semblait à première vue et que, pour des raisons que nous ne pouvons ici détailler, le programme prévu par l'université de Saransk et l'Institut des Humanités était raisonnable.

13. S. Kovaleva travaille surtout sur le carélien, mais maitrise également le finnois. Le finnois était, avec partiellement le vepse, la langue de travail de J.L. Léonard sur place ; pour K. Djordjević Léonard, c'était le russe.

14. Les "activistes" vepsophiles ont ainsi, dans les années 1990, fait entrer le vepse pour la première fois à l'Université, créé le journal Kodima, lancé le programme des nids de langues, créé des associations culturelles, amorcé une intense activité de traduction et de production scientifique et littéraire. Ces activités ont été décrites dans Djordjević Léonard, 2013.

15. C'est ainsi que les nomme Laura Siragusa dans sa thèse de doctorat en anthropologie sociale, intitulée Vepsian Language: Speaking and Writing Heritage Language in Villages and Cities, et soutenue en 2012 à l'université d'Aberdeen, en Écosse. Comme le hasard fait parfois bien les choses, il se trouve que nous avons eu l'occasion de nous entretenir avec L. Siragusa à Petrozavodsk même, où elle était venue donner une conférence durant notre séjour, et d'échanger ainsi nos points de vue de «chercheurs occidentaux » quant aux effets de l'aménagement linguistique « de par en bas » ou « à mi-palier » sur la situation sociolinguistique du vepse.

16. Notre questionnaire sociolinguistique était inspiré par celui utilisé pour le projet PEPS «Les langues et vous» (université de Poitiers \& université de Paris 3, 2010-2011, cf. Léonard \& Jagueneau, 2013, 2014), que nous avons adapté et traduit en russe. Ce questionnaire est destiné précisément aux protagonistes de la revitalisation, et s'organise autour des axes suivants : libre arbitre interne et externe, expériences décisives, sphère privée versus sphère publique et centres d'intérêt, conformément à la méthodologie des récits de vie élaborée par J.P. Roos (1985). Les différentes questions ont été modifiées au fur et à mesure des entretiens biographiques, si bien que les derniers entretiens ont été menés sans questionnaires. Pour une version du questionnaire initial, cf. Léonard et Djordjević Léonard, à paraître.

17. Cf. par exemple, et pour ne citer que quelques références majeures, Zaiceva \& Mullonen, 2007, Zajceva \& Žukova, 2009, Zajceva, 2010, Strogal'ščikova, 2008.

18. L'Institut de langue, littérature et histoire de Carélie propose, sur son site, un certain nombre de ces publications en accès libre.

http://illhportal.krc.karelia.ru

19. Cette maison d'édition, créée en 1991, publie cinq revues en carélien, finnois et vepse: Karjalan Sanomat (« НОВОСТИ КАРЕЛИИ »), Ота тиа (« РОДНАЯ ЗЕМЛЯ »), Кodima ( РОДНАЯ ЗЕМЛЯ »), Carelia (« КАРЕЛИЯ ») et Кіріnä (« ИСКОРКА »).

20. On distingue trois régions vepses : la zone vepse nord couvre la rive sud-ouest du lac Onega, la zone centrale s'étend sur une partie des oblasts de Leningrad et de 
Vologda, la région méridionale est celle située au sud-est de Saint-Pétersbourg (oblast de Leningrad).

21. Pour un aperçu de ces témoignages, cf. Djordjević Léonard, 2014, à paraître.

22. Cf. pour un aperçu de la situation sociolinguistique dans les domaines administratif, éducatif, informatif et culturel, Djordjević Léonard, 2013.

23. Anecdote rapportée par N. Zajceva dans l'entretien réalisé le 24/04/2013, à Petrozavodsk.

24. La zone vepse nord comporte les villages suivants : ШоКША / Šokš, ШЁЛтОзЕРО / Šoutjärv', РЫБРЕКА / Kaleig, КАСКЕСРУчЕЙ / Kaskez, ВЕХРУ́чЕЙ / Vehkoi, ДРУГАЯ РЕКА / Toižeg.

25. Légende urbaine rapportée par Z Strogal'ščikova dans l'entretien réalisé le 24/04/2013, à Petrozavodsk.

26. http://kaino.kotus.fi/sanat/vepsa/index.php? $\mathrm{t}=$ hae\&hakusana $=\&$ merkitys=sammakko\&pitaja $=$ La base de données donne effectivement les formes suivantes : löts, -un ; löpshǟne -häst, -

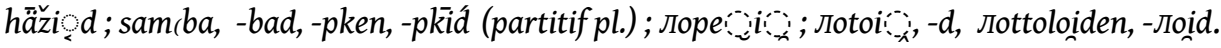

27. http://kaino.kotus.fi/sanat/vepsa/index.php?t=hae\&hakusana=\&merkitys $=\mathrm{y} \%$ C3\%B6lepakko\&pitaja $=$

28. On s'en doute, certes, mais ce détail mérite d'être rappelé : vepse et finnois ne sont pas mutuellement intelligibles sans un apprentissage préalable. Nous avons pu le constater à de nombreuses reprises aussi bien avec des locuteurs vepses ruraux qu'urbains, y compris avec une institutrice de vepse du lycée finno-ougrien de Petrozavodsk.

29. Cf. http://vk.com/club25641591

Le réseau VKontakte est l'équivalent russe de Facebook.

30. Nous avons, à cette occasion, enregistré 1 h 20 de discussions, chants et poèmes en vepse et en russe.

31. Cf. Djordjević Léonard, 2013, 2014 à paraitre, Léonard \& Djordjević Léonard, 2014 à paraître, Léonard, 2013, 2014. 


\section{INDEX}

Index géographique : Alsace, Amérique centrale, Balkans, Barents (mer de), Bordeaux, Carélie (République), Catalogne, Finlande, France, Helsinki, Italie, Kiži (lac), Ladva, Léningrad (oblast'), Mordovie (République), Moscou, Murmansk, Onega, Pays basque, Pays de Galles, Petrozavodsk, Arhangel'sk (oblast'), Rybreka, Saint-Pétersbourg, Saransk, Solovki (îles), Šokša, Tampere, Volga (vallée de la), Vologda

Keywords : Assimilation, fieldwork, language planning, revitalisation, Sociolinguistics, twentyfirst century (beginning), Carelian, English, Finnish, Livvi, Lude, Russian, Veps, Estonians, Finns Thèmes : sociolinguistique

Mots-clés : aménagement linguistique, assimilation, travaux de terrain, revitalisation nomsmotscles Estoniens, Finlandais, Vepses

disciplines anglais, carélien, finnois, livvi, lude, russe, vepse 